\title{
SECURED TEXT MESSAGE TRANSMISSION WITH IMPLEMENTATION OF CONCATENATED CFB CRYPTOGRAPHIC ALGORITHM
}

\author{
Dr.S.Saravana Kumar ${ }^{1}$, Ms R.Dharani ${ }^{2}$, Aniruth Sabapathy ${ }^{3}$, D.Nirmaldas ${ }^{4}$, \\ S.Srikanth ${ }^{5}$
}

\begin{abstract}
In the present simulated system, text message transmission has been secured with concatenated implementation of Cipher Feedback(CFB) cryptographic algorithm. It is anticipated from the numerical results that the pre-ZF channel equalization based MIMO OFDM wireless communication system outperforms in QAM digital modulation and BCH channel coding under AWGN and Raleigh fading channels .In Pre-MMSE/pre-ZF channel equalization scheme, the system shows comparatively worst performance in convolutional channel coding scheme with QAM/QPSK digital modulation. It has been observed from the present study that the system performance deteriorates with increase in noise power as compared to signal power. study of a secured MIMO Orthogonal Frequency-Division Multiplexing wireless communication system with implementation of two pre channel equalization techniques such as Pre-Minimum Mean Square Error (Pre-MMSE) and PreZero Forcing(Pre-ZF)under QPSK and QAM digital modulations.
\end{abstract}

\section{KEYWORDS}

MIMO-OFDM, Pre-MMSE, Pre-ZF, ECB and CFB cryptographic algorithm, Bit Error rate(BER), $A W G N$ and Raleigh fading channels.

\section{INTRODUCTION}

Multiple-Input, Multiple-Output (MIMO) technique using multiple antennas at both the transmit and receive ends has become one of the most important paradigms for the deployment of existing and emerging wireless communications systems. MIMO constitutes a cost effective approach to high-throughput wireless communications. MIMOs are capable of supporting significantly higher data rates than the Universal Mobile Telecommunications System (UMTS) and the High-Speed Downlink Packet Access (HSDPA) based 3G networks. MIMO OFDM(Orthogonal Frequency-Division Multiplexing) communication systems have shown increased capacity, coverage and achievable reliability with the aid of MIMO techniques. The OFDM has emerged as a successful air-interface multicarrier digital modulation technique advocated by many European standards, such as Digital Audio Broadcasting (DAB), Digital Video Broadcasting for Terrestrial television (DVB-T), Digital Video Broadcasting for Handheld terminals (DVB-H), Wireless Local Area Networks (WLANs) and Broadband Radio

Sundarapandian et al. (Eds): CoNeCo,WiMo, NLP, CRYPSIS, ICAIT, ICDIP, ITCSE, CS \& IT 07, pp. 463-472, 2012. (C) CS \& IT-CSCP 2012

DOI : $10.5121 /$ csit.2012.2445 
Access Networks (BRANs).High-speed cellular and WLAN standards (i.e., 4G cellular including WiMAX and LTE, and 802.11a,g,n) have migrated to OFDM, which offers higher spectral efficiency and performance With MIMO signal processing and wider band channels, it becomes possible to increase peak bit rates to the range of $100 \mathrm{Mb} / \mathrm{s}$ in both LTE and WiMAX systems. The MIMO based OFDM technologies have been used in WLAN systems to achieve significantly higher bit rates. The $802.11 \mathrm{n}$ standard has a peak bit rate of $300 \mathrm{Mb} / \mathrm{s}$ using OFDM with higher order adaptive modulation and MIMO along with multiple channel techniques[1-3].

\section{MATHEMATICAL MODEL}

In our presently considered secured spatially multiplexed MIMO OFDM wireless communication system, various Pre channel equalization schemes, Electronic Codebook (ECB) and Cipher Feedback (CFB) cryptographic algorithms have been used. A brief description is given below.

\subsection{Pre-Channel Equalization}

In pre channel equalization scheme, pre equalization is represented by a pre-equalizer weight matrix in complex form( $\mathrm{W} \in \mathrm{C} 2 \times 2)$ and the precoded digitally modulated complex symbol vector $\mathrm{x} \in \mathrm{C} 2 \mathrm{x} 1$ can be expressed as

$$
x=W \tilde{x}
$$

$\tilde{x}$ is the original symbol vector for transmission. In case of zero-forcing (ZF) equalization employment, the corresponding weight matrix (assuming that the channel matrix $\mathrm{H}$ issquare) is given as

$$
W_{z F}=\beta H
$$

Where $\beta$ is a constant to meet the total transmitted power constraint after pre-equalization and it is given with two transmitting antenna( $\mathrm{NT}=2)$ as

$$
\beta-\sqrt{\frac{n_{\tau}}{\operatorname{Tr}\left(H^{-1}\left(H^{-1}\right)^{H}\right)}}
$$

To compensate for the effect of amplification by a factor of $\beta$ at the transmitter, the received signal must be divided by b via automatic gain control (AGC) at the receiver, The received signal $\mathrm{y}$ is given by

$$
\begin{aligned}
& y=\frac{1}{\beta}\left(H W_{z F} \tilde{x}+z\right) \\
& \tilde{x}+\frac{1}{\beta} z \\
& ={ }_{\beta}^{1}\left(H \beta H^{-1} \tilde{x} \quad \mid:\right) \\
& =\tilde{x}+\hat{z}
\end{aligned}
$$


Other than ZF pre-equalization, MMSE pre-equalization can also be used. In this case, theweight matrix is given as

$$
\begin{aligned}
W_{M M S E}=\beta \times \underset{\text { arg } \min }{w} E\left\{\left\|\beta^{-1}(H W \tilde{x}+z)-\tilde{x}\right\|^{2}\right\} & \\
& =\beta \times H^{H}\left(H H^{H}+\frac{\sigma_{z}^{2}}{\sigma_{x}^{2}} I\right)^{-1}
\end{aligned}
$$

Where, the constant $\beta$ is used again to meet up the total transmitted power constraint.

It is calculated by Equation (3) replacing $\mathrm{H}^{-1}$ with $H^{H}\left(H H^{H}+\frac{\sigma_{z}^{2}}{\sigma_{x}^{2}} I\right)^{-1}$. It is noted that the preequalization scheme on the transmitter side outperforms the receiver-side equalization. It is attributed to the fact that the receiver-side equalization suffers from noise enhancement in the course of equalization[4].

\subsection{Cryptographic algorithm}

Cryptography is synonymous with encryption and used exclusively on providing confidentiality of messages. With the advancement of information technology, a great emphasis has been given to provide confidentiality, integrity and authentication for secured data transmission. In Symmetric Key Cryptography, there are two types of symmetric key cipher; namely, stream ciphers and block ciphers. With stream ciphers, data is encrypted one digit (bit or byte) at a time. Stream ciphers approximate one-time pad ciphers (also known as Vernam ciphers). In contrast, a block cipher processes fixed-length groups of bits. A block of plaintext symbols are encrypted to create a block ciphertext of the same size [6].

The most widely used encryption scheme is based on the Data Encryption Standard (DES) adopted in 1977 by the National Bureau of Standards. The DES scheme is essentially a block cipher technique that uses certainnumber of bit blocks. In Electronic Codebook (ECB) block cipher cryptographic scheme, we have divided the whole message into several blocks of each size 64 bits. Each block of plaintext is encrypted using the same key of size 64 bits. In CipherFeedback (CFB) cryptographic scheme, the input is processed with64 bits at a time. Preceding ciphertext is used as input to the encryption algorithm to produce pseudorandom output, which is XORed with plaintext to produce next unit of ciphertext [7].

\section{COMMUNICATION SYSTEM MODEL}

A simulated single -user 2 x 2 spatially multiplexed MIMO OFDM wireless communication system as depicted in Figure 1 utilizes two pre channel equalization schemes.In such a communication system, the text message is encrypted doubly with concatenation of Electronic Codebook (ECB) and Cipher Feedback ( CFB) cryptographic algorithm. The encrypted text message is channel encoded using each of the three channel coding scheme ( $1 / 2$-rated convolutional, $\mathrm{CRC}$ an $\mathrm{BCH}$ ) and interleaved for minimization of burst errors. The interleaved bits are digitally modulated using two types of digital modulations such as Quadrature Phase Shift Keying (QPSK) and Quadrature Amplitude (QAM) [8,9]. The complex digitally modulated symbols are spatially multiplexed using Alamouti's space -time block coding (STBC), an efficient transmit diversity scheme [10]. The outputs of the Space- time block 
encoder are sent up into two serial to parallel converter. The serial to parallely (S/P) converted complex data symbols are fed into each of the two OFDM modulator with 1024 sub carriers which performs an IFFT on each OFDM block of length 1024 followed by a parallel -to- serial conversion. A cyclic prefix (CP) of length Lcp (0.1*1024) containing a copy of the last Lcp samples of the parallel -to- serial converted output of the 1024-point IFFT is then prepended. The $\mathrm{CP}$ is essentially a guard interval which serves to eliminate interference between OFDM symbols.

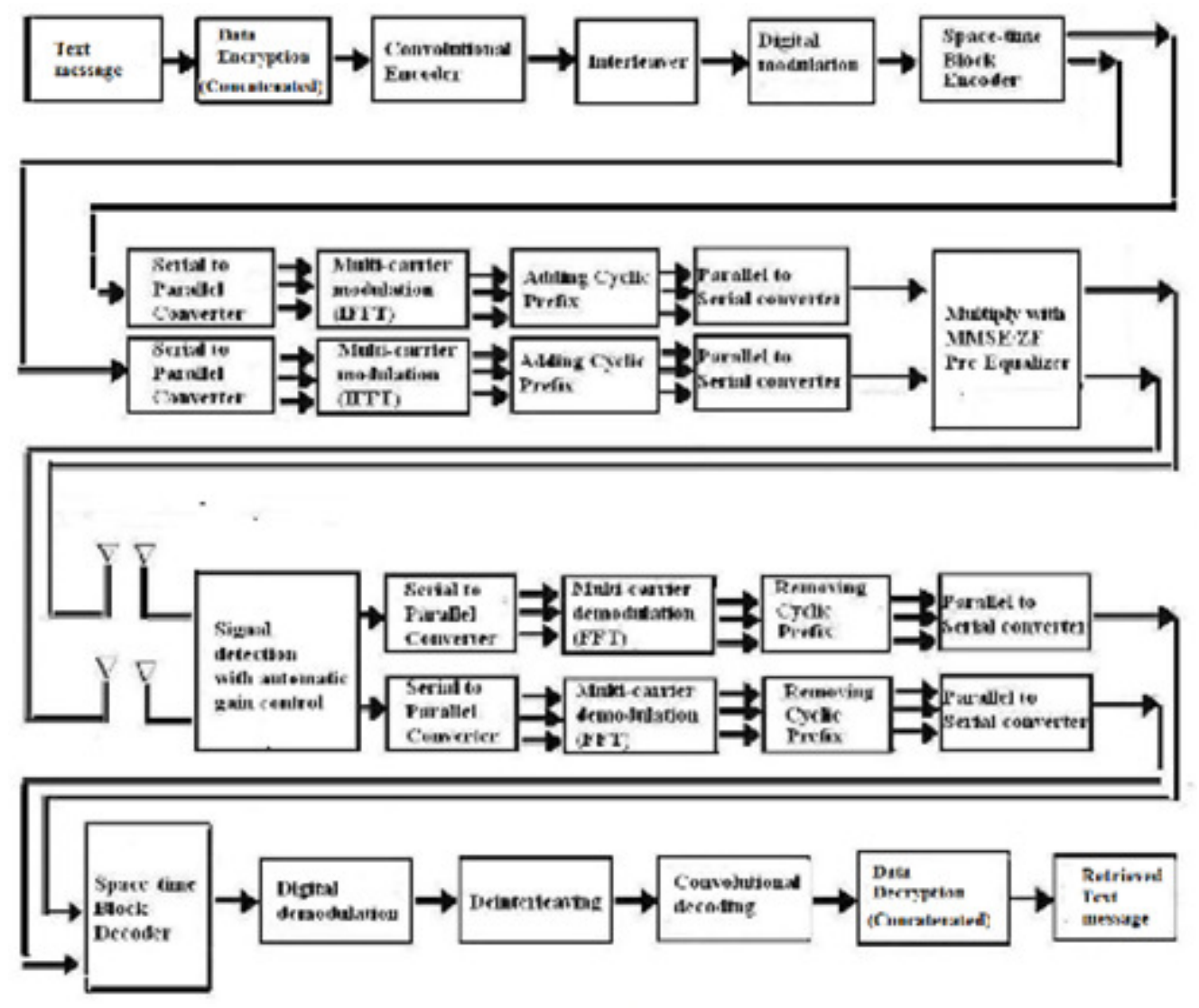

Figure 1: Block diagram of a Pre-Channel Equalization based secured MIMO OFDM wireless communication system

However, the resulting OFDM symbols of length 1024+ Lcp are weighted with pre equalization channel coefficients prior to lunching from the two transmitting antenna. In case of post channel equalization, no pre-channel equalization operation is performed. However, in receiving section, the signals are detected and passed through automatic gain controlling section to compensate the effect of amplification at the transmitter. The detected signals are subsequently sent up to the serial to parallel (S/P) converter and fed into OFDM demodulator which performs FFT operation on each OFDM block. The FFT operated OFDM blocked signal are processed with cyclic prefix removing scheme and are undergone from parallel to serial conversion and are fed into Space time block decoder. Its output in complex symbols are digitally demodulated, deinterleaved, channel decoded and decrypted doubly to recover the transmitted text message. 


\section{RESULT AND DISCUSSION}

The present simulation based study has been made for MIMO OFDM wireless communication system in consideration with various parameters presented in Table 1.

Table 1: Summary of the simulated model parameters

\begin{tabular}{|l|l|}
\hline Text message(Converted into & 1024 \\
\hline Channel Coding & 1 2-rated Convolutional, CRC and BCH Channel \\
\hline Modulation & QPSK and QAM \\
\hline No of OFDM sub-carriers & 1024 \\
\hline Cryptographic algorithm & Electronic Codebook(ECB) and Cipher Feedback(CFB) \\
\hline Channel Equalization Scheme & $\begin{array}{l}\text { Pre-Mean Square error (Pre-MMSE) and Pre-Zero- } \\
\text { Forcing (Pre-ZF) }\end{array}$ \\
\hline CP length & 103 symbols \\
\hline Channel & AWGN and Rayleigh \\
\hline Signal to noise ratio, SNR & 0 to10 dB \\
\hline
\end{tabular}

We have conducted computer simulations to observe the impact of pre and post channel equalization schemes on the BER performance of the secured MIMO OFDM wireless communication system based on the parameters given in Table 1. It is assumed that the channel state information (CSI) is available at the transmitter side and the fading process is approximately constant during one OFDM block length.

The graphical illustrations presented in Figure 2 through Figure 5 show system performance comparison with implementation of pre-MMSE and pre-ZF based Channel equalization schemes under QPSK and QAM digital modulations. In all cases, it is noticeable that Pre-ZF based channel equalization with $\mathrm{BCH}$ channel coding schemes improves the system performance. In Figure 2, it is observable that the system performance is well discriminable less than three different channel coding schemes. For a typically assumed SNR value of $2 \mathrm{~dB}$, the BER values are 0.4609 and 0.2485 in case of Convolutional and $\mathrm{BCH}$ channel coding schemes under QPSK and Pre-MMSE and viz., the system achieves a gain of $2.68 \mathrm{~dB}$ in $\mathrm{BCH}$ as compared to $1 / 2$-rated Convolutional. The system shows well defined performance over a large examined SNR values under the situation of three channel coding schemes and QAM and Pre- MMSE (Figure 3). At a SNR value of $2 \mathrm{~dB}$, the system performance is improved by $3.15 \mathrm{~dB}$ for BER values of 0.1673 and 0.3454 in case of $\mathrm{BCH}$ as compared to $1 / 2$-rated Convolutional. 


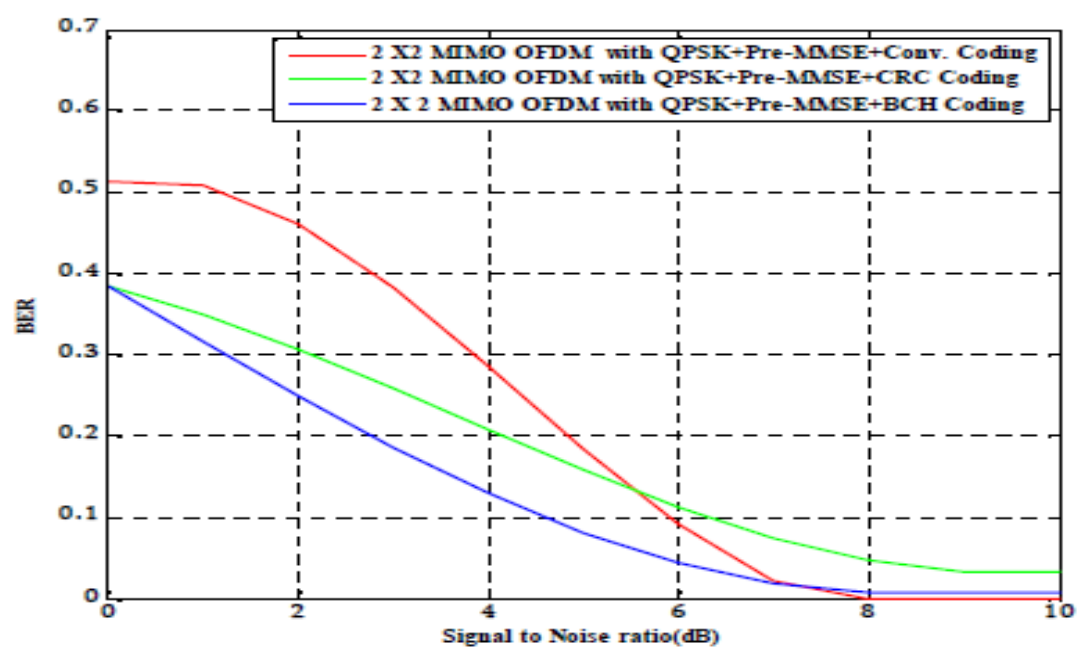

Figure 2 : BER Performance of a secured MIMO OFDM wireless communication with implementation of different Channel coding, Pre-MMSE channel equalization and QPSK digital modulation schemes.



Figure 3 : BER Performance of a secured MIMO OFDM wireless communication with implementation of different Channel coding, Pre-MMSE channel equalization and QAM digital modulation schemes.

In Figure 4, and Figure 5, it is observable that the rate of system performance improvement with increase in SNR values. is comparatively higher in Convolutional coding as compared to CRC and $\mathrm{BCH}$. In Figure 4 and Figure 5, the system performance improvement is found to have values of 2.76dB (BERs: 0.2363 and 0.4461) and 3.40dB(BERs: 0.1565 and 0.3425) at a typically assumed SNR value of $2 \mathrm{~dB}$ in case of most satisfactory and worst system performance. 




Figure 4 : BER Performance of a secured MIMO OFDM wireless communication with implementation of different Channel coding, Pre-ZF channel equalization and QPSK digital modulation schemes.



Figure 5: BER Performance of a secured MIMO OFDM wireless communication with implementation of different Channel coding, Pre-ZF channel equalization and QAM digital modulation schemes.

In Figure 6, a BER comparison has been made for the system in identical channel coding and different digital modulation and pre-channel equalization schemes. It is remarkable that over a significant SNR value area, the system shows quite satisfactory performance in QAM, Pre-ZF and $\mathrm{BCH}$ schemes. Under identical implementation of channel coding $(\mathrm{BCH})$ scheme, the system shows worst performance in QPSK and Pre-MMSE. Asystem performance improvement of $2.01 \mathrm{~dB}$ is achieved at SNR value of $2 \mathrm{~dB}$ in QAM, Pre-ZF and BCH schemes as compared to QAM, Pre-MMSE and BCH(BERs: 0.1565 and 0.2485). 


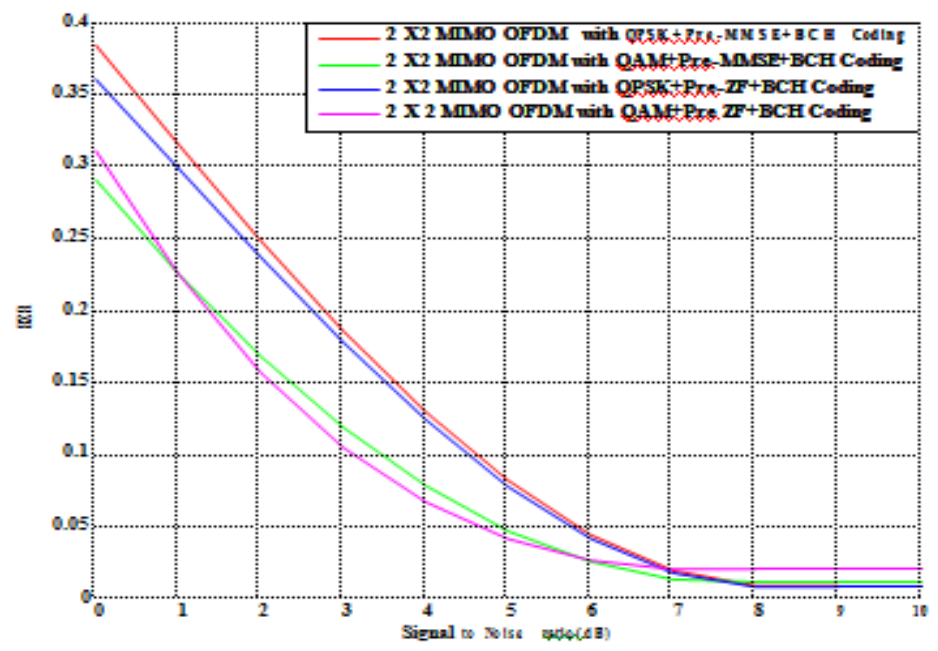

Figure 6 : BER Performance Comparison of the MIMO OFDM wireless communication system for different Pre channel equalization and digital modulation schemes under $\mathrm{BCH}$ channel coding scheme.

In Figure 7, the transmitted, doubly encrypted and retrieved text messages at $4 \mathrm{~dB}$ and $6 \mathrm{~dB}$ have been presented under implementation of QAM, Pre-ZF and $\mathrm{BCH}$ channel coding schemes. The estimated BER values are found to have values of 0.0166 and 0.000 .

Multiple-input multiple-output systems have attracted considerable attention due to the linear growth of the system capacity.

(a)

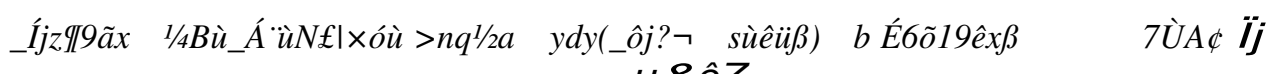

\section{$u \& \hat{e} Z$}

yQØÀa_jò ,êZßIV+\$\#?o $\partial \times B \mid$.

(b)

Multiple-input multipLeoõôx\}ô óystemshave attracted considerable attention due to the linear growth of the system0s 1 !cmpy.

(c)

Multiple-input multiple-output systems have attracted considerable attention due to the linear growth of the system capacity.

(d)

Figure 7 : Presented text message in various forms in a MIMO OFDM wireless communication system , (a) Transmitted, (b) Doubly Encrypted (c) Retrieved message at 4 dB (d) Retrieved message at $6 \mathrm{~dB}$. Red marks indicate noise contamination 


\section{CONCLUSIONS}

In our present study, we have studied the performance of a Convolutionally encoded $2 \times 2$ spatially multiplexed MIMO-OFDM wireless communication system adopting various digital modulations, channel coding and Pre channel Equalization schemes. A range of system performance results highlights the impact of a simplified digital modulation, Pre channel Equalization (signal detection) and channel coding techniques. In the context of system performance, it can be concluded that the implementation of QAM digital modulation technique with implemented Pre-ZF channel equalization and $\mathrm{BCH}$ channel coding schemes in FEC encoded MIMO-OFDM wireless communication system provides satisfactory performance in retrieving the transmitted message of the sender.

\section{REFERENCES}

[1] LajosHanzo, YosefAkhtman, Li Wang and Ming Jiang, (2011), " MIMO-OFDM for LTE, Wi-Fi and WiMAX , Coherent versus Non-coherent and Cooperative Turbo-transceivers", John Wiley and Sons, Ltd, United Kingdom.

[2] DipankarRaychaudhuri and Narayan B. Mandayam ,(2012), " Frontiers of Wireless and Mobile Communications", Proceedings of the IEEE vol. 100, no. 4, pp. 828-840.

[3] Alain Sibille, Claude Oestges and Alberto Zanella, (2011), "MIMO From Theory to Implementation" , Elsevier Inc., United Kingdom

[4] Yong Soo Cho, Jaekwon Kim, Won Young Yang, Chung G. Kang, (2010), "MIMO-OFDM Wireless Communications with MATLAB", John Wiley and Sons (Asia) Pte Limited, Singapore.

[5] Lin BaiandJinho Choi(2012), "Low Complexity MIMO Detection", Springer Science and Business Media, LLC ,New York, USA

[6] Alan Holt and Chi-Yu Huang (2010), "802.11 Wireless Networks Security and Analysis", Springer-Verlag London Limited, New York.

[7] William Stallings(2005),"Cryptography and Network Security Principles and Practices", Fourth Edition, Prentice Hall Publisher.

[8] Theodore S Rappaport(2001) Wireless Communications: Principles and Practice, Second Edition, Prentice Hall, Upper Saddle River, New Jersey, USA

[9] Goldsmith, Andrea, 2005 :Wireless Communications, First Edition, Cambridge University Press,United Kingdom

[10] Siavash M. Alamouti (1998), A Simple Transmit Diversity Technique For Wireless Communications, IEEE Journal on Select areas in Communications, vol.16, no.8, pp.1451-1458 


\section{AUTHORS}

Dr S.SARAVANAKUMAR has more than 10 years of teaching and research experience. He did his Postgraduate in $\mathrm{ME}$ in Computer Science and Engineering at Bharath engineering college, Chennai, and PhD in Computer Science and Engineering at Bharath University, Chennai. He occupied various positions as Lecturer, Senior Lecturer, Assistant Professor and Associate Professor and HOD. He has published more than 35 research papers in High Impact factor International Journal, National and International conferences and visited many countries like Taiwan, Bangkok and Singapore. He has guiding a number of research scholars in the area Adhoc Network, ANN, Security in Sensor Networks, Mobile Database and Data Mining under Bharath University Chennai, Sathayabama University and Bharathiyar University.

Ms. R. Dharani has more than 8 years of teaching experience. She has occupied various positions as Lecturer, Senior Lecturer, Assistant Professor (Grade-I) . She has published more than 25 research papers in High Impact factor International Journal, National and International conferences and visited many countries like Taiwan, Bangkok and Singapore. He has guiding a number of research scholars in the area Adhoc Network, ANN, Security in Sensor Networks, Mobile Database and Data Mining under Bharath University Chennai, Sathayabama University and Bharathiyar University.

G. Aniruth Sabapathy is a final year student pursuing his B.tech Information Technology, Panimalar institute of technology, Chennai. He has attended several conferences and National level symposium. He has submitted papers on cloud computing, peer to peer systems and search engine optimization. His domain of Interest are Database management and systems, web technology, web designing, search engine optimization. He has created a search engine optimization website (www.directionswitch.com).

Nirmal das.D is a final year student pursuing his B.Tech Information Technology, in Panimalar institute of technology, Chennai. He has attended several conferences and National level symposium. He has submitted papers on peer to peer systems and search engine optimization. His domain of Interest in the area Adhoc Network, ANN, Security in Sensor Networks, Mobile Database and Data Mining.

S.Srikanth is a final year student pursuing his B.Tech Information Technology, in Panimalar institute of technology, Chennai. He has attended several conferences and National level symposium. He has submitted papers on cloud computing, peer to peer systems and search engine optimization. His domain of Interest are Database management and systems, web technology, web designing, search engine optimization
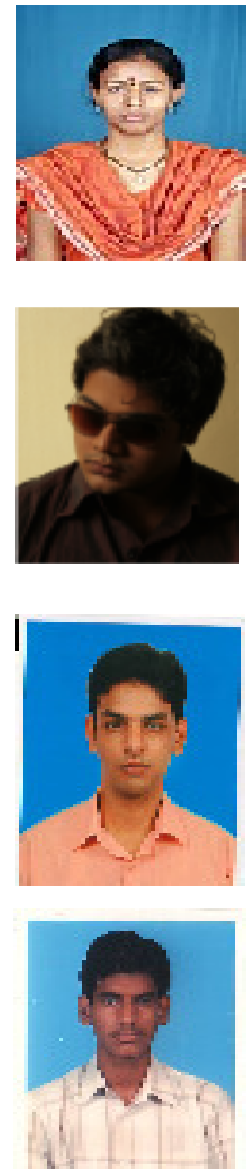